\title{
Gamma-ray burst afterglows and their implications
}

\author{
P. Mészáros \\ Department of Astronomy \& Astrophysics, Pennsylvania State University, University Park, PA 16802, U.S.A. \\ e-mail: nnp@astro.psu.edu
}

Received December 29, 1998; accepted March 10; 1999

\begin{abstract}
The discovery of X-ray, optical and radio afterglows of GRBs provides an important tool for understanding these sources. Most current models envisage GRB as arising in a cataclysmic stellar event leading to a relativistically expanding fireball, where particle acceleration at shocks lead to nonthermal radiation. The predictions of this scenario are in substantial agreement with the bulk of the observations. In addition, the data show a diversity of finer structure behavior, which is providing constraints for more detailed models. Current issues of interest are the implications of the beaming for the energetics, the afterglow time structure, dependence on progenitor system, and the role of the environment.
\end{abstract}

Key words: gamma-ray bursts — shocks waves

\section{Introduction: Simple "standard" afterglows}

One can understand the dynamics of the afterglows of GRB in a fairly simple manner, independently of any uncertainties about the progenitor systems, using a relativistic generalization of the method used to model supernova remnants. The simplest hypothesis is that the afterglow is due to a relativistic expanding blast wave, which decelerates as time goes on (Mészáros \& Rees 1997a; earlier simplified discussions were given by Katz 1994b; Paczyński \& Rhoads 1993; Rees \& Mészáros 1992). The complex time structure of some bursts suggests that the central trigger may continue for up to $100 \mathrm{~s}$. However, at much later times all memory of the initial time structure would be lost: essentially all that matters is how much energy and momentum has been injected; the injection can be regarded as instantaneous in the context of the much longer afterglow. Detailed calculations and predictions from such a model (Mészáros \& Rees 1997a) preceded the observations of the first afterglow detected, GRB 970228 (Costa et al. 1997; van Paradijs et al. 1997).

The simplest spherical afterglow model produces a three-segment power law spectrum with two breaks.
At low frequencies there is a steeply rising synchrotron self-absorbed spectrum up to a self-absorption break $\nu_{\mathrm{a}}$, followed by a $+1 / 3$ energy index spectrum up to the synchrotron break $\nu_{\mathrm{m}}$ corresponding to the minimum energy $\gamma_{\mathrm{m}}$ of the power-law accelerated electrons, and then a $-(p-1) / 2$ energy spectrum above this break, for electrons in the adiabatic regime (where $\gamma^{-p}$ is the electron energy distribution above $\gamma_{\mathrm{m}}$ ). A fourth segment and a third break is expected at energies where the electron cooling time becomes short compared to the expansion time, with a spectral slope $-p / 2$ above that. With this third "cooling" break $\nu_{\mathrm{b}}$, first calculated in Mészáros et al. 1998 and more explicitly detailed in Sari et al. 1998, one has what has come to be called the simple "standard" model of GRB afterglows. This assumes spherical symmetry (also valid for a jet whose opening angle $\theta_{\mathrm{j}} \gtrsim \Gamma^{-1}$ ). As the remnant expands the photon spectrum moves to lower frequencies, and the flux in a given band decays as a power law in time, whose index can change as breaks move through it.

The standard model assumes an impulsive energy input lasting much less than the observed $\gamma$-ray pulse, characterized by a single energy and bulk Lorentz factor value (delta or top-hat function). Estimates for the time needed for the expansion to become non-relativistic could then be $\lesssim$ month (Vietri 1997a), especially if there is an initial radiative regime $\Gamma \propto r^{-3}$. However, even when electron radiative times are shorter than the expansion time, it is unclear whether such a regime occurs, as it would require strong electron-proton coupling (Mészáros et al. 1998). The standard spherical model can be straightforwardly generalized to the case where the energy is assumed to be channeled initially into a solid angle $\Omega_{\mathrm{j}}<4 \pi$. In this case (Rhoads 1997a, 1997b) there is a faster decay of $\Gamma$ after sideways expansion sets in, and a decrease in the brightness is expected after the edges of the jet become visible, when $\Gamma$ drops below $\Omega_{j}^{-1 / 2}$. A calculation using the usual scaling laws for a single central line of sight leads then to a steepening of the light curve. 


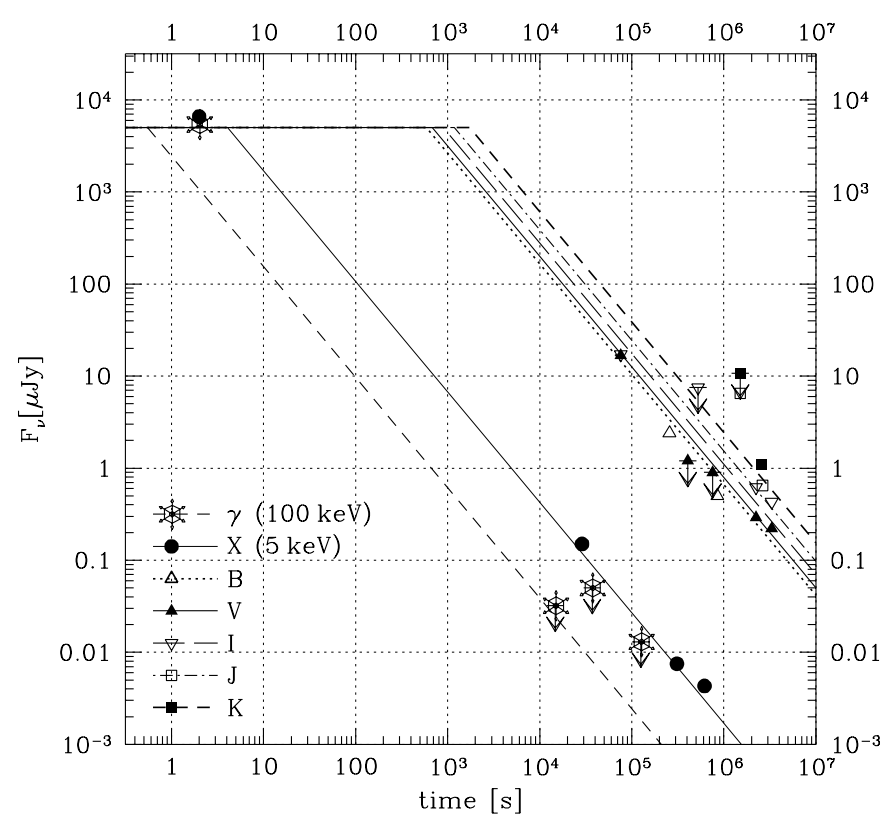

Fig. 1. Light-curves of GRB 970228, compared to the blast wave model predictions of Mészáros \& Rees 1998a (from Wijers et al. 1997)

The simple standard model has been remarkably successful at explaining the gross features of GRB 970228, GRB 970508, etc. (Wijers et al. 1997; Tavani 1997; Waxman 1997; Reichart 1997). Spectra at different wavebands and times have been extrapolated according to the simple standard model time dependence to get spectral snapshots at a fixed time (Waxman 1997; Wijers \& Galama 1998), allowing fits for the different physical parameters of the burst and environment, e.g. the total energy $E$, the magnetic and electron-proton coupling parameters $\epsilon_{\mathrm{B}}$ and $\epsilon_{\mathrm{e}}$ and the external density $n_{\mathrm{o}}$. In GRB 971214 (Ramaprakash et al. 1998), a similar analysis and the lack of a break in the late light curve of GRB 971214 could be interpreted as indicating that the burst (including its early gamma-ray stage) was isotropic, leading to an (isotropic) energy estimate of $10^{53.5}$ ergs. Such large energy outputs, whether beamed or not, are quite possible in either NS-NS, NS-BH mergers (Mészáros \& Rees 1997b) or in hypernova/collapsar models (Paczyński 1998; Popham et al. 1998), using MHD extraction of the spin energy of a disrupted torus and/or a central fast spinning BH. However, it is worth stressing that what these snapshot fits constrain is only the energy per solid angle (Mészáros et al. 1998b). The expectation of a break after only some weeks or months (e.g., due to $\Gamma$ dropping either below a few, or below $\Omega_{\mathrm{j}}^{-1 / 2}$ ) is based upon the simple impulsive (angle-independent delta or top-hat function) energy input approximation. The latter is useful, but departures from it would be quite natural, and certainly not surprising. As discussed below, it would be premature to conclude at present that there are any significant constraints on the anisotropy of the outflow.

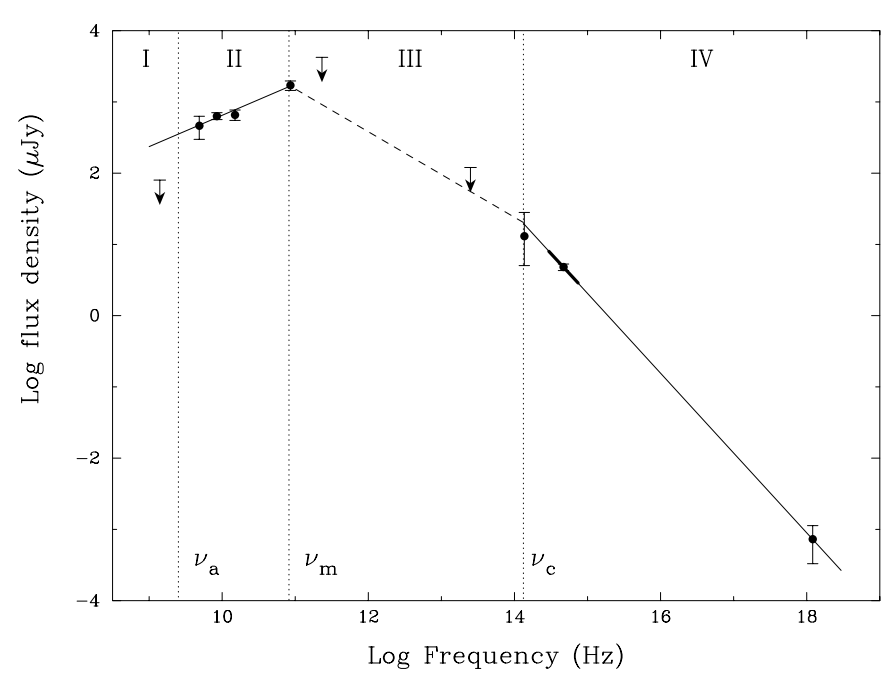

Fig. 2. Snapshot spectrum of GRB 970508 at $t=12$ days and standard afterglow model fit (after Wijers \& Galama 1998)

\section{2. "Post-standard" afterglow models}

In a realistic situation, one could expect any of several fairly natural departures from the simple standard model to occur. The first one is that departures from a delta top-hat approximation (e.g. having more energy emitted with lower Lorentz factors at later times, still shorter than the gamma-ray pulse duration) would drastically extend the afterglow lifetime in the relativistic regime, by providing a late "energy refreshment" to the blast wave on time scales comparable to the afterglow time scale (Rees \& Mészáros 1998). The transition to the $\Gamma<\theta_{\mathrm{j}}^{-1}$ regime occurring at $\Gamma \sim$ few could then occur as late as six months to more than a year after the outburst, depending on details of the brief energy input.

Another important effect is that the emitting region seen by the observer resembles a ring (Waxman 1997b; Panaitescu \& Mészáros 1998b; Sari 1998). A numerical integration over angles (Panaitescu \& Mészáros 1998d) shows that the sideways expansion effects are not so drastic as inferred from the scaling laws for the material along the central-angle line of sight. This is because even though the flux from the head-on part of the remnant decreases faster, this is more than compensated by the increased emission measure from sweeping up external matter over a larger angle, and by the fact that the extra radiation, arising at larger angles, arrives later and re-fills the steeper light curve. Thus, the sideways expansion (even for a simple impulsive injection) actually mitigates the flux decay, rather than accelerating it. Combined with the possibility of an extended relativistic phase due to nonuniform injection, and the fact that numerical angle integrations show that any steepening would occur over factors $\sim 2-3$ in time, one must conclude that we $d o$ not yet have significant evidence for whether the outflow is jet-like or not. 

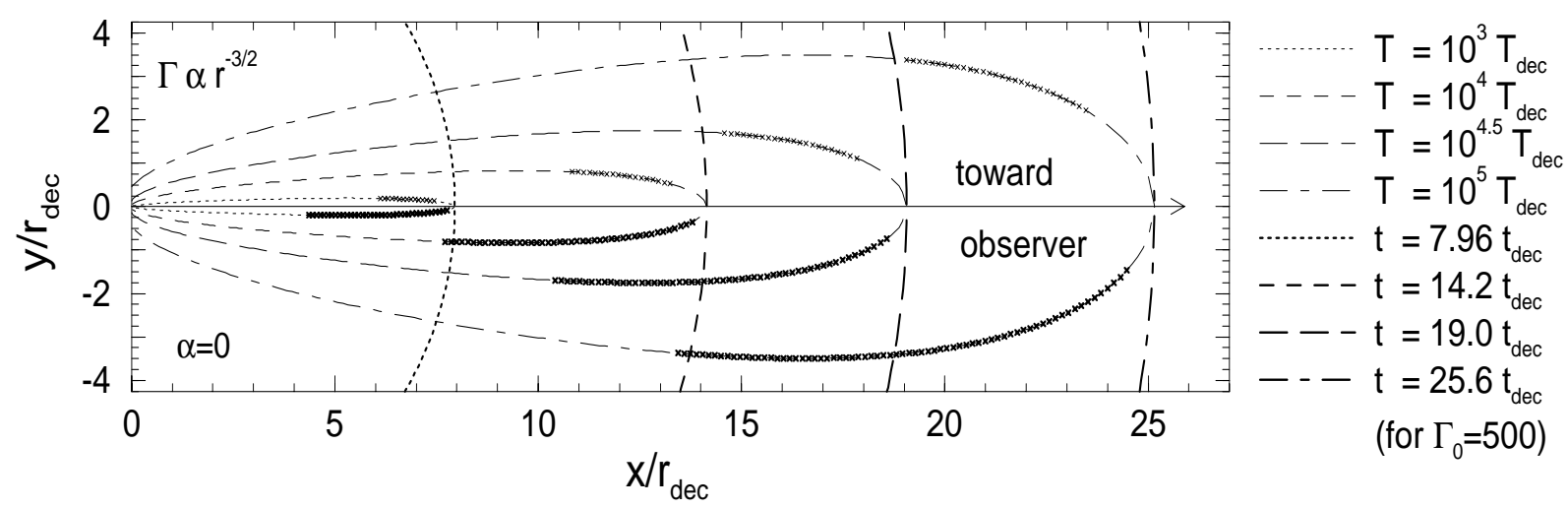

Fig. 3. Ring-like equal-arrival time $T$ surfaces of an afterglow, based on Panaitescu \& Mészáros (1998d)

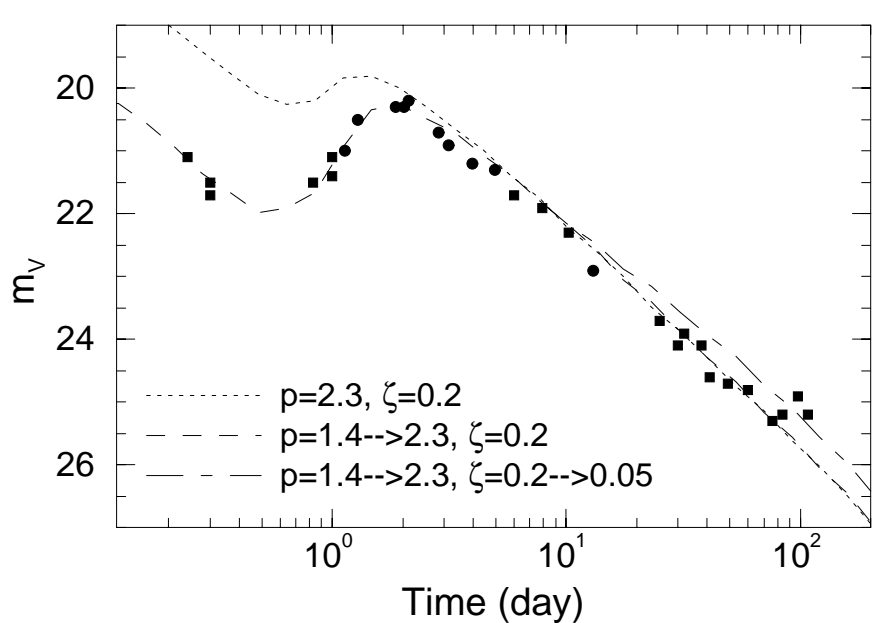

Fig. 4. Optical light-curve of GRB 970508, fitted with a nonuniform injection model (Panaitescu et al. 1998)

One expects afterglows to show a significant amount of diversity. This is expected both because of a possible spread in the total energies (or energies per solid angle as seen by a given observer), a possible spread or changes in the injected bulk Lorentz factors, and also from the fact that GRB may be going off in very different environments. The angular dependence of the outflow, and the radial dependence of the density of the external environment can have a marked effect on the time dependence of the observable afterglow quantities (Mészáros et al. 1998). So do any changes of the bulk Lorentz factor and energy output during even a brief energy release episode (Rees \& Mészáros 1998).

Strong evidence for departures from the simple standard model is provided by, e.g., sharp rises or humps in the light curves followed by a renewed decay, as in GRB 970508 (Pedersen et al. 1998; Piro et al. 1998). Detailed time-dependent model fits (Panaitescu et al. 1998) to the X-ray, optical and radio light curves of GRB 970228 and GRB 970508 show that, in order to explain the humps, a non-uniform injection or an anisotropic outflow is required. These fits indicate that the shock physics may be a function of the shock strength (e.g. the electron index $p$, injection fraction $\zeta$ and/or $\epsilon_{\mathrm{b}}, \epsilon_{\mathrm{e}}$ change in time), and also indicate that dust absorption is needed to simultaneously fit the X-ray and optical fluxes. The effects of beaming (outflow within a limited range of solid angles) can be significant (Panaitescu \& Mészáros 1998c), but are coupled with other effects, and a careful analysis is needed to disentangle them.

Spectral signatures, such as atomic edges and lines, may be expected both from the outflowing ejecta (Mészáros \& Rees 1998a) and from the external medium (Perna \& Loeb 1998; Mészáros \& Rees 1998b; Bisnovatyi-Kogan \& Timokhin 1997) in the X-ray and optical spectrum of afterglows. These may be used as diagnostics for the outflow Lorentz factor, or as alternative measures of the GRB redshift. An interesting prediction (Mészáros \& Rees 1998b; see also Ghisellini et al. 1998; Böttcher et al. 1998) is that the presence of a measurable Fe K- $\alpha$ emission line could be a diagnostic of a hypernova, since in this case one can expect a massive envelope at a radius comparable to a light-day where $\tau_{\mathrm{T}} \lesssim 1$, capable of reprocessing the X-ray continuum by recombination and fluorescence.

The location of the afterglow relative to the host galaxy center can provide clues both for the nature of the progenitor and for the external density encountered by the fireball. A hypernova model would be expected to occur inside a galaxy, in fact inside a high density $\left(n_{\mathrm{o}}>10^{3}-10^{5}\right)$. Some bursts are definitely inside the projected image of the host galaxy, and some also show evidence for a dense medium at least in front of the afterglow (Owen et al. 1998). On the other hand, for a number of bursts there are strong constraints from the lack of a detectable, even faint, host galaxy (Schaefer 1998). In NS-NS mergers one would expect a BH plus debris torus system and roughly the same total energy as in a hypernova model, but the mean distance traveled from birth is of order several Kpc (Bloom et al. 1998), leading to a burst presumably in a less dense environment. The fits of Wijers \& Galama 1998 to the observational data on GRB 970508 and GRB 971214 in fact suggest external densities in the range of $n_{\mathrm{o}}=0.04-0.4 \mathrm{~cm}^{-1}$, which would 
be more typical of a tenuous interstellar medium (however, Reichart \& Lamb 1998 report a fit for GRB 980329 with $n_{\mathrm{o}} \sim 10^{4} \mathrm{~cm}^{-3}$ ). These could arise within the volume of the galaxy, but on average one would expect as many GRB inside as outside. This is based on an estimate of the mean NS-NS merger time of $10^{8}$ years; other estimated merger times (e.g. $10^{7}$ years, van den Heuvel 1992) would give a burst much closer to the birth site. BH-NS mergers would also occur in timescales $\lesssim 10^{7}$ years, and would be expected to give bursts well inside the host galaxy (Bloom et al. 1998).

\section{Conclusions}

The blast wave model of gamma-ray burst afterglows has proved quite robust in providing a consistent overall interpretation of the major features of these objects at various frequencies. The "standard model" of afterglows, involving four spectral slopes and three breaks, is quite useful in understanding "snaphsot" multiwavelength spectra of afterglows. However, the constraints on the angleintegrated energy, especially at $\gamma$-ray energies, are not strong, and beaming effects remain uncertain. Some caution is required in interpreting the observations on the basis of the simple standard model. For instance, if one integrates the flux over all angles visible to the observer, the contributions from different angles lead to a considerable rounding-off of the spectral shoulders, so that breaks cannot be easily located unless the spectral sampling is dense and continuous, both in frequency and in time. Some of the observed light curves with humps, e.g. in GRB 970508, require "post-standard" model features (i.e. beyond those assumed in the standard model), such as either non-uniform injection episodes or anisotropic outflows. Time-dependent multiwavelength fits of this and other bursts also seem to indicate that the parameters characterizing the shock physics change with time. A relatively brief $(1-100 \mathrm{~s})$, probably modulated energy input appears the likeliest interpretation for most bursts. This can provide an explanation both for the highly variable $\gamma$-ray light curves and for late glitches in the afterglow decays.

There has been significant progress in understanding how gamma-ray bursts can arise in fireballs produced by brief events depositing a large amount of energy in a small volume, and in deriving the generic properties of the ensuing long wavelength afterglows. There still remain a number of mysteries, especially concerning the identity of their progenitors, the nature of the triggering mechanism, the transport of the energy and the time scales involved. However, independently of the details of the gammaray burst central engine, even if beaming reduces their total energy requirements, these objects are the most extreme phenomena that we know about in high energy astrophysics, and may provide useful beacons for probing the universe at $z \gtrsim 5$. With new experiments coming on-line in the near future, there is every prospect for continued and vigorous developments both in the observational and theoretical understanding of these fascinating objects.

Acknowledgements. I am grateful to Martin Rees for stimulating collaborations on this subject, as well as to Ralph Wijers, Hara Papathanassiou and Alin Panaitescu. This research is supported in part by NASA NAG5-2857

\section{References}

Bisnovatyi-Kogan G., Timokhin A., 1997, Astr. Rep. 41, 423 Bloom J., Sigurdsson S., Pols O., 1998, MNRAS (in press) (astro-ph/9805222)

Böttcher M., et al., 1998, astro-ph/9809156

Costa E., et al., 1997, Nat 387, 783

Ghisellini G., et al., 1998, astro-ph/9808156

Katz J., 1994b, ApJ 432, L107

Kulkarni S., et al., 1998, Nat 393, 35

Mészáros P., Rees M.J., 1997a, ApJ 476, 232

Mészáros P., Rees M.J., 1997b, ApJ 482, L29

Mészáros P., Rees M.J., 1998a, ApJL 502, L105

Mészáros P., Rees M.J., 1998b, MNRAS 299, L10 (astro-ph/9806183)

Mészáros P., Rees M.J., Wijers R., 1998, ApJ 499, 301 (astro-ph/9709273)

Mészáros P., Rees M.J., Wijers R., 1998b, New Astron (in press) (astro-ph/9808106)

Metzger M., et al., 1997, Nat 387, 878

Owen A., et al., 1998, A\&A (in press) (astro-ph/9809356)

Paczyński B., Rhoads J., 1993, ApJ 418, L5

Paczyński B., 1998, ApJ 494, L45

Panaitescu A., Mészáros P., 1998b, ApJ 493, L31

Panaitescu A., Mészáros P., 1998c, ApJ (submitted) (astro-ph/9806016)

Panaitescu A., Mészáros P., Rees M.J., 1998, ApJ 503, 314

Pedersen H., et al., 1998, ApJ 496, 311

Perna R., Loeb A., 1998, ApJL 503, L135

Piro L., et al., 1998, A\&A 331, L41

Popham R., Woosley S., Fryer C., 1998, ApJ (submitted) (astro-ph/9807028)

Ramprakash A.N., et al., 1998, Nat 393, 38

Rees M.J., Mészáros P., 1992, MNRAS 258, P41

Rees M.J., Mészáros P., 1998, ApJL 496, L1

Reichart D., 1997, ApJ 485, L57

Reichart D., Lamb D.Q., 1999 (this issue)

Rhoads J., 1997a, ApJ 487, L1

Rhoads J., 1997b (preprint) (also this issue)

Sari R., 1998, ApJ 494, L49

Sari R., Piran T., Narayan R., 1998, ApJ 497, L17 (astro-ph/9712005)

Schaefer B.E., 1998, ApJ (in press) (astro-ph/9810424)

Tavani M., 1997, ApJ 483, L87

Thompson C., 1994, MNRAS 270, 480

van den Heuvel E., in X-ray Binaries and Recycled Pulsars,

Heuvel E.v.d., Rappaport S. (eds.). Kluwer 1992, p. 233

van Paradijs J., et al., 1997, Nat 386, 686

Vietri M., 1997a, ApJ 478, L9

Waxman E., 1997, ApJL 485, L5

Waxman E., 1997b, ApJL 489, L33

Wijers R., Galama T., 1998, ApJ (submitted) (astro-ph/9805341)

Wijers R., Rees M.J., Mészáros P., 1997, MNRAS 288, L51 